\title{
Min-Type Control Strategy of a DC-DC Synchronous Boost Converter
}

\author{
Antonino Sferlazza, Member, IEEE, Carolina Albea-Sanchez, Luis Martínez-Salamero, Senior Member, \\ IEEE, Germain García, Corinne Alonso
}

\begin{abstract}
This paper presents the analysis and design of a min-type strategy to control a synchronous boost converter in continuous conduction mode. The strategy uses a nonlinear switching surface to establish the change of topology in the converter and is analyzed by means of a sliding-mode control approach. Subsequently, the min-type strategy is modified by a hybrid control formulation, which introduces a hysteresis width and a dwell-time to obtain a finite switching frequency in the start-up and steady-state respectively. The hybrid control formulation is implemented digitally by means of a microprocessor which processes the samples of inductor current and capacitor voltage to provide the control signal that activates the power switch. Experimental results in a prototype validate the proposed control strategy and show its potential in transient time and steady-state.
\end{abstract}

Index Terms-Synchronous boost converter, min-type control, hybrid control, sliding-mode control.

\section{INTRODUCTION}

Non-minimum phase converters are traditionally controlled in industrial applications by a cascade regulation scheme [1]. The inner loop controls an inductor current while the outer loop regulates the output voltage by giving an appropriate reference to the inner loop. The cascade approach is usually implemented with analogue circuits, which in some cases can be based on specific commercial chips [2]. The implementations mainly differ in the way they process the inductor current, which is actually regulated by controlling the dynamic behavior of some of its indexes such as peak, valley or average values [3]. They also differ in the type of modulator, which can be Pulse Width Modulator (PWM) or hysteretic. Regarding PWM schemes, diverse approaches can be also found, the main difference being the type of periodic signal they use.

Moreover, control digital of DC-DC switching converters has been progressively moving in the last years from academic research to industrial applications, this being favored by the rapid development and extended use of high performance

Manuscript received September 14, 2018; revised October 23, 2018 , January 09, 2019, and February 18, 2019; accepted March 08, 2018.

Antonino Sferlazza is with the Department of Engineering, University of Palermo, Palermo, Italy (e-mail: antonino.sferlazza@unipa.it).

Luis Martínez-Salamero is with the Group of Automatic Control and Industrial Electronics, Rovira i Virgili University, Tarragona, Spain (email: luis.martinez@urv.cat).

Carolina Albea-Sanchez, Germain García and Corinne Alonso are with the University of Toulouse III (Université Paul Sabatier), and with the LAAS CNRS, Toulouse, France (e-mail: calbea laas. fr, alonsoclaas.fr). digital processors. In this context, the cascade control of a boost converter has also been digitally implemented with both PWM or hysteresis approaches, the inner control loop being based either on current prediction to avoid the continuous sampling of the inductor current [4], [5], [6], [7], [8], [9], [10], [11], [12], [13] or on discrete-time sliding-mode approach [14]. In most cases, the inner loop establishes the transition to OFF state by comparing an index of the increasing inductor current waveform with an upper reference while the transition to ON state is carried out either symmetrically, i.e. an index of the decreasing inductor current waveform is compared with a lower reference, or by the action of an external periodical clock signal.

This paper tackles the digital implementation of the inner loop by means of a min-type approach. In the proposal, what establishes the transition between states is the difference between two indexes $M_{0}$ and $M_{1}$, which is calculated by the digital processor. Each index is associated to a calculation of the state vector trajectory from given initial conditions to the equilibrium point, which is defined by the desired output voltage in a clear-cut contrast with the conventional approach in which the desired inductor current establishes the operating point of the inner loop. Indeed, in other control techniques, (e.g. sliding mode control, PI control) the inner loop is driven only by the inductor current error, without considering the output voltage error, which is often controlled by a second loop. The min-type approach drives the inner loop by the state error, which contains output voltage and inductor current, and thus, directly controls the whole trajectory in the phaseplane voltage-current. Unlike conventional control designs, it is worth mentioning that the conception of a min-type control implicitly guarantees stability of the inner loop, without requiring any additional proof. Moreover, the computation of the above mentioned index $M_{0}$ and $M_{1}$ requires the solution of an optimization problem that minimizes the tracking error.

The main antecedent of this approach was formulated in [15] and enhanced in [16], [17] in the context of switched affine systems control design, where it was demonstrated that the control law optimizing the trajectory from zero-initial conditions to the equilibrium point was an on-off switching law leading to a variable switching frequency. The parallelism between the formulation in [15] and Sliding-Mode Control (SMC) for a particular linear switching surface was explored in [18], in which the switching surface coefficients were calculated off-line using the min-type control formulation with the aim of minimizing the waste of energy in the transition 
from zero initial conditions to the equilibrium point. The equivalence between SMC and min-type control is valid on the assumption of infinite switching frequency. This theoretical constraint has prompted some reports to deal with the introduction of a dwell time between successive commutations in order to limit the maximum switching frequency [19], [20]. In that context, a preliminary work to attain a trade-off between the switching frequency and the level of optimality was published in [21] by formulating the problem in terms of hybrid dynamical systems [22], [23]. All the mentioned papers on either min-type or hybrid control lack of experimental verification, which reduces their potential applications.

This paper tackles the problem of implementing a min-type control of a synchronous boost converter using the nonlinear control switching surface proposed in [15] and subsequently used in the hybrid control formulation reported in [21]. An additional goal is to present the advantages of such switching surface in the converter start-up and explore its potential use for output voltage regulation. The problem of switching frequency limitation is also analyzed by interpreting the effect of the mentioned hybrid control formulation during start-up and by adapting the resulting control for digital implementation.

The rest of the paper is organized as follows. Section II reviews the notion of equilibrium point locus of the boost converter by showing the analytical expression of an ellipse relating all the possible pairs of inductor current and capacitor voltage values in steady-state. The min-type control strategy based on a nonlinear switching surface is interpreted in terms of sliding-mode control is shown in Section III. The min-type control is subsequently modified by a hybrid control approach in Section IV in order to obtain a finite switching frequency. Details about implementation are given in Section V, and experimental results and comparisons with SMC and PI control are given in Section VI. Conclusions are in Section VII.

\section{EQUILIBRIUM POINT LOCUS}

The synchronous boost converter depicted in Fig. 1 can be described by the set of parameters $\left(V_{i n}, L, C, R_{0}, R_{L}\right)$, where $V_{i n}$ is the input voltage, $L$ and $C$ are the inductance and capacitance respectively, $R_{0}$ is the load resistance and $R_{L}$ is the parasitic resistance of the inductor. The dynamic behavior of the converter in continuous conduction mode can be described in compact form as follows:

$$
\begin{aligned}
\frac{d i_{L}}{d t} & =-\frac{R_{L}}{L} i_{L}-\frac{v_{C}}{L}(1-u)+\frac{V_{i n}}{L}, \\
\frac{d v_{C}}{d t} & =\frac{i_{L}}{C}(1-u)-\frac{1}{C R_{0}} v_{C},
\end{aligned}
$$

where $i_{L}$ is the inductor current, $v_{C}$ is the capacitor voltage, and $u$ is a control binary signal $(\bar{u}=1-u)$ such that $u=1$ $(\bar{u}=0)$ during $T_{\text {on }}$ (interval of energy absorption from the input source) and $u=0(\bar{u}=1)$ during $T_{\text {off }}$ (interval of energy transfer to the output load). Note that the converter shown in Fig. 1 is bidirectional because it allows the flow of power in both directions, from the input to the load and vice versa, therefore the inductor current can assume negative values. For this reason, the converter is analyzed only in continuousconduction mode because the discontinuous mode is automat-

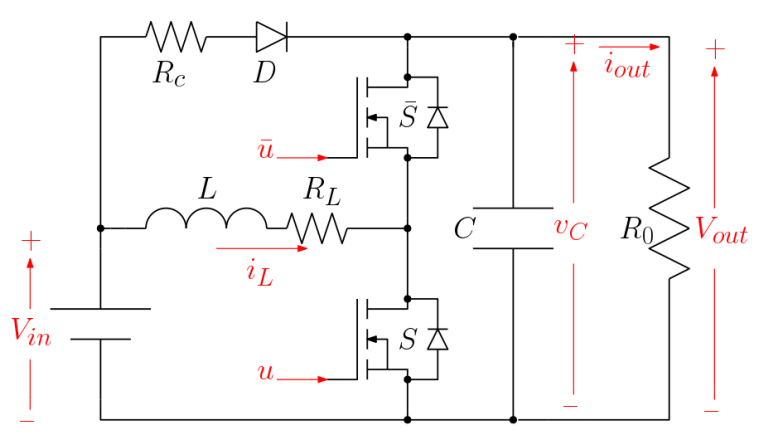

Figure 1. Synchronous boost converter.

Table I

\begin{tabular}{|c|c|c|}
\hline SYMBOL & VALUe & UNIT \\
\hline$V_{\text {in }}$ & {$\left[\begin{array}{lll}24 & 40\end{array}\right]$} & $\mathrm{V}$ \\
\hline$L$ & 470 & $\mu \mathrm{H}$ \\
\hline$R_{L}$ & 3 & $\mathrm{~m} \Omega$ \\
\hline$C$, & 20 & $\mu \mathrm{F}$ \\
\hline$R_{0}$ & {$\left[\begin{array}{ll}100 & 150\end{array}\right]$} & $\Omega$ \\
\hline
\end{tabular}

CIRCUIT PARAMETERS VALUES

ically not allowed. On the other hand, diode $D$ charges the capacitor at start-up avoiding a large inrush current. Note that this diode is not active when the output voltage becomes higher than the input one. Moreover, the charging resistance $R_{c}$ does not affect the efficiency of the system, because during normal operation the output voltage is always higher than the input one, and diode $D$ blocks the current to flow through $R_{c}$. In order to see the effect of the auxiliary diode $D$ that attenuates the inrush current, the reader is addressed to [24, Figs. 8-9].

The Equilibrium Point Locus (EPL) of the switching converter is the set of points in the plane $i_{L}-v_{C}$ that the converter state variables can take in steady-state. In the converter considered here, the EPL is given by the following equation [24]:

$$
v_{C}^{2}+R_{L} R_{0} i_{L}^{2}-R_{0} V_{i n} i_{L}=0 .
$$

Equivalently:

$$
\frac{\left(i_{L}-\frac{c}{2 b}\right)^{2}}{\frac{c^{2}}{4 b^{2}}}+\frac{v_{C}^{2}}{\frac{c^{2}}{4 b^{2}}}=1,
$$

where $b=R_{L} R_{0}$ and $c=R_{L} V_{i n}$. It can be observed that the EPL given by (3) is an ellipse of center $\left(\frac{c}{2 b}, 0\right)$, horizontal axis $\frac{c}{b}$ and vertical axis $\frac{c}{\sqrt{b}}$, in the plane $i_{L}-v_{C}$.

\section{Min-TyPE CONTROL INTERPRETATION}

The min-type control strategy proposed in [15] based on a nonlinear switching surface can be expressed as follows:

$$
\sigma(x)=\arg \min _{u \in\{0,1\}}\left(x-X_{E}\right)^{\top} P\left(A_{u} x+B V_{i n}\right) .
$$

Matrices $A_{u}$ and $B$ relate the state vector $x=\left(\begin{array}{ll}i_{L} & v_{C}\end{array}\right)^{\top}$ and its time derivative, i.e. $\dot{x}=A_{u} x+B V_{i n}$. Specifically, $B=\left[\begin{array}{ll}\frac{1}{L} & 0\end{array}\right]^{\top}$, and matrices $A_{u}$ are:

$$
\begin{aligned}
& A_{0}=\left[\begin{array}{cc}
-\frac{R_{L}}{L} & -\frac{1}{L} \\
\frac{1}{C} & -\frac{1}{R_{0} C}
\end{array}\right], \quad \text { for } u=0\left(T_{\text {off }} \text { interval }\right), \\
& A_{1}=\left[\begin{array}{cc}
-\frac{R_{L}}{L} & 0 \\
0 & -\frac{1}{R_{0} C}
\end{array}\right], \quad \text { for } u=1\left(T_{\text {on }} \text { interval }\right),
\end{aligned}
$$


$X_{E}\left(I_{E}, V_{E}\right)$ is the desired equilibrium point in EPL, and $P$ is a symmetric positive-definite matrix given by:

$$
P=\left[\begin{array}{ll}
\alpha & \beta \\
\beta & \gamma
\end{array}\right]
$$

and satisfying:

$$
A_{u}^{\top} P+P A_{u}+2 Q \leq 0, \quad u=0,1,
$$

where $Q$ is an auxiliary symmetric positive-definite matrix. In order to find a strictly positive definite matrix $P$ such that (8) is satisfied, the following optimization problem is solved:

$$
\begin{aligned}
P^{*}= & \arg \min _{P=P^{\top}} \operatorname{trace}(P), \quad \text { subject to: } \\
& A_{u}^{\top} P+P A_{u}+2 Q \leq 0, \quad \forall u \in\{0,1\}, \\
& P \geq I_{2},
\end{aligned}
$$

Note the inequality $P \geq I_{2}$ has been used instead of $P>$ 0 . This choice has been made to avoid numerical problems and solutions that lead to small values of $P$ because its trace is minimized. In any case any matrix $P$ solution to (9) is also a solution to the problem with $P>0$ because it has relaxed constraints. The algorithm to find a solution $P$ to the optimization problem (9) can be found in [25, Section IV].

The cost function $J$ of the transition from the initial state $x(0)$ to the equilibrium point $X_{E}$ is bounded and given by:

$$
\begin{aligned}
J=\int_{0}^{\infty}\left(x(t)-X_{E}\right)^{\top} Q\left(x(t)-X_{E}\right) d t< \\
\left(x(0)-X_{E}\right)^{\top} P\left(x(0)-X_{E}\right) .
\end{aligned}
$$

Note that $X_{E}=\left[\begin{array}{ll}I_{E} & V_{E}\end{array}\right]^{\top}$, where $I_{E}$ and $V_{E}$ are the mean values of the inductor current $i_{L}$ and output voltage $V_{\text {out }}$ at steady-state, respectively. Moreover, the minimization of the trace of $P$, as given in (9), leads to the minimization of the cost function $J$.

The first term in (10) is the time integral of the weighted square error of the state vector trajectory with respect to the desired equilibrium point while the second term is the weighted Euclidean distance between the start point and the equilibrium point. The interpretation of (4) results in two possible cases:

$$
\begin{aligned}
& \sigma(x)=0 \Leftrightarrow\left(x-X_{E}\right)^{\top} P\left(A_{0} x+B V_{i n}\right)=M_{0} \text { is minimum, } \\
& \sigma(x)=1 \Leftrightarrow\left(x-X_{E}\right)^{\top} P\left(A_{1} x+B V_{i n}\right)=M_{1} \text { is minimum. }
\end{aligned}
$$

If we define the switching surface $S(x)$ as $S(x)=M_{1}-M_{0}$ we will obtain:

$$
S(x)=\left(x-X_{E}\right)^{\top} P\left(A_{1}-A_{0}\right) x .
$$

Therefore, the control law (4) can be interpreted as follows:

$$
u=0 \text { if } S(x)>0, u=1 \text { if } S(x)<0 .
$$

Introducing the expressions of $X_{E}, P, A_{0}$ and $A_{1}$ in (12) yields:

$$
\begin{aligned}
S(x)= & \frac{\beta v_{C}^{2}}{L}-\frac{\beta i_{L}^{2}}{C}+\left(\frac{\alpha}{L}-\frac{\gamma}{C}\right) i_{L} v_{C} \\
& -\left(\frac{\alpha I_{E}}{L}+\frac{\beta V_{E}}{L}\right) v_{C}+\left(\frac{\beta I_{E}}{C}+\frac{\gamma V_{E}}{C}\right) i_{L} .
\end{aligned}
$$

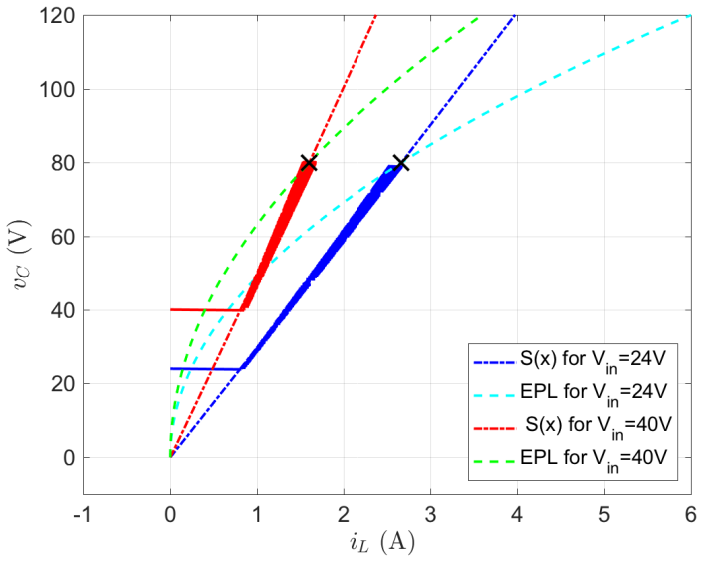

Figure 2. Simulation of the converter start-up for $V_{i n}=24 \mathrm{~V}$ (blue trajectory) and $V_{i n}=40 \mathrm{~V}$ (red trajectory) and min-type control with nonlinear switching surface $S(x)$.

It can be observed that expression (14) is a conic curve that passes through the origin of the plane $i_{L}-v_{C}$ and the equilibrium point $X_{E}\left(I_{E}, V_{E}\right)$.

Fig. 2 shows a Matlab ${ }^{\circledR}$ simulation of the converter startup in the plane $i_{L}-v_{C}$ for the set of parameters $C, L$ and $R_{L}$ shown in Tab. I, $R_{0}=100 \Omega, V_{E}=80 \mathrm{~V}$, and two possible input voltages, i.e. $V_{i n}=24 \mathrm{~V}$ (blue trajectory) and $V_{i n}=40 \mathrm{~V}$ (red trajectory). Matrix $P$ used in the simulation is the solution of the optimization problem (9), with $Q=\left[\begin{array}{cc}R_{L} & 0 \\ 0 & \frac{\rho}{R_{0}}\end{array}\right]$ and $\rho=1000$ :

$$
P^{*}=\left[\begin{array}{ll}
\alpha^{*} & \beta^{*} \\
\beta^{*} & \gamma^{*}
\end{array}\right]=\left[\begin{array}{cc}
2.3108 & -0.0097 \\
-0.0097 & 1.0001
\end{array}\right] .
$$

In both cases the initial voltage of the output capacitor coincides with the input voltage to reduce the inrush current as demonstrated in [24].

It can be observed that the proposed switching surface results in a negligible inrush current irrespective of the input voltage value. Also, it has to be pointed out that the coordinate $V_{E}$ is the same in both cases while the coordinate $I_{E}$ changes according to $I_{E}=\frac{V_{E}^{2}}{R_{0} V_{i n}}$, neglecting the losses in resistor $R_{L}$.

Note that Eq.s (13) and (14) have been introduced to give an interpretation in terms of sliding mode control. Nevertheless, there are many differences because the proposed control technique is formulated using a new framework: the hybrid dynamical systems introduced in [22], and explained later in Section IV. Note that the control variable $u$ comes from the arg-min strategy (4), which is different from the sliding mode paradigm. Moreover, in strategy (4) there is embedded a second optimization problem related to the selection of matrix $P$. In particular, matrix $Q$ can be arbitrarily chosen in order to provide any performance level of the converter by weighting suitably both output voltage and inductor current errors. For example, if $\rho$ is chosen such that $\rho / R_{0}$ is greater than $R_{L}$ (which are the elements of matrix $Q$ given before in Eq. (15)), this will imply that we give more weight to the voltage than to the current. Therefore, it can be expected to obtain an output voltage faster convergence, at the expense of a larger 
current ripple. After the choice of $Q$, matrix $P$ is selected by solving the optimization problem (9), which leads to the minimization of the cost function $J$. On the other hand, it can be observed that in sliding mode control there are not optimization problems explicitly considered.

\section{HYBRID CONTROL APPROACH}

A practical implementation of the control law (13) requires the introduction of hysteresis around the switching surface [26] in order to obtain finite switching frequency required by the power devices. The hysteresis function can be implemented analogically [27] or digitally by using some microcontroller peripherals as reported in [28]. Nonetheless, the resulting dynamics and the sliding dynamics are strictly speaking the same only when the hysteresis width tends to zero. A preliminary attempt to indirectly introduce a hysteresis for switched affine systems was explored in [21] under the optics of a hybrid control formulation. As a matter of fact system (1a)-(1b) can be considered as a switching system, i.e. a differential equation whose right-hand side is chosen from a family of functions based on a switching signal $u$. This kind of system can be contextualized in the hybrid system framework. A hybrid dynamical system (see [22] for further details) exhibits a combination of continuous-time and discrete-time dynamics and can be represented as:

$$
\mathcal{H}= \begin{cases}\dot{z}=f(z), & z \in \mathcal{C}, \\ z^{+}=g(z), & z \in \mathcal{D} .\end{cases}
$$

As suggested by (16), the state of the hybrid system $z$ can evolve according to a differential equation $\dot{z}=f(z)$ (where the vector field $f$ is called flow map) if the state $z$ belongs to the flow set $\mathcal{C}$, or it can change according to a difference equation $z^{+}=g(z)$ (where the vector field $g$ is called jump map) if the state $z$ belongs to the jump set $\mathcal{D}$. The notation $z^{+}$ is the value of the state after the jump, i.e. the value assumed by $g(z)$ when $z$ belongs to $\mathcal{D}$. According with this formalism the system (1a)-(1b) can be written in the form (16) where:

$$
z=\left[\begin{array}{c}
i_{L} \\
v_{c} \\
u
\end{array}\right], \quad f=\left[\begin{array}{c}
-\frac{R_{L}}{L} i_{L}-\frac{v_{C}}{L}(1-u)+\frac{V_{i n}}{L} \\
-\frac{i_{L}}{C}(1-u)-\frac{1}{C R_{0}} v_{C} \\
0
\end{array}\right], \quad g=\left[\begin{array}{c}
i_{L} \\
v_{c} \\
1-u
\end{array}\right] .
$$

Note that $u$ has been considered as a further state variable with dynamics $\dot{u}=0$ during flow.

In order to fix the dynamical behavior of the converter, the flow set $\mathcal{C}$ and the jump set $\mathcal{D}$ have to be suitably chosen. In [21] the sets $\mathcal{C}$ and $\mathcal{D}$ were defined as follows:

$$
\begin{aligned}
\mathcal{C}:=\left\{(x, u):\left(x-X_{E}\right)^{\top} P\left(A_{u} x+B V_{i n}\right)\right. \\
\left.\leq \eta\left(x-X_{E}\right)^{\top} Q\left(x-X_{E}\right)\right\} \\
\mathcal{D}:=\left\{(x, u):\left(x-X_{E}\right)^{\top} P\left(A_{u} x+B V_{i n}\right)\right. \\
\left.>\eta\left(x-X_{E}\right)^{\top} Q\left(x-X_{E}\right)\right\}
\end{aligned}
$$

where $0<\eta \leq 1$, and $Q=\left[\begin{array}{cc}R_{L} & 0 \\ 0 & \frac{\rho}{R_{0}}\end{array}\right], \rho$ being an auxiliary positive parameter. Note that $\eta$ is strictly greater than zero to avoid Zeno behaviours. The switching strategy involved in (18)-(19) consists in remaining in the current converter topology if (18) is accomplished or changing the topology if (19) applies. It is worth mentioning that it was also demonstrated in [21, Theorem 1] that the switching law (18)-(19) leads to an equilibrium point $X_{E}$, which is uniformly globally asymptotically stable.

Now, we interpret the control law (18)-(19) by studying the resulting state vector trajectory in the plane $i_{L}-v_{C}$ as in the case of the min-type control (13) illustrated in Fig. 2.

Defining $S_{0}(x)$ and $S_{1}(x)$ as:

$S_{0}(x)=\left(x-X_{E}\right)^{\top} P\left(A_{0} x+B V_{i n}\right)+\eta\left(x-X_{E}\right)^{\top} Q\left(x-X_{E}\right)$,

$S_{1}(x)=\left(x-X_{E}\right)^{\top} P\left(A_{1} x+B V_{i n}\right)+\eta\left(x-X_{E}\right)^{\top} Q\left(x-X_{E}\right)$,

the corresponding expressions of $S_{0}(x)$ and $S_{1}(x)$ in terms of state variables and system parameters are given by:

$$
\begin{aligned}
S_{1}(x)= & \eta R_{L}\left(i_{L}-I_{E}\right)^{2}+\eta \frac{\rho}{R_{0}}\left(v_{C}-V_{E}\right)^{2} \\
& +\left(\alpha \frac{V_{i n}-R_{L} i_{L}}{L}-\frac{\beta v_{C}}{C R_{0}}\right)\left(i_{L}-I_{E}\right) \\
& +\left(\beta \frac{V_{i n}-R_{L} i_{L}}{L}-\frac{\gamma v_{C}}{C R_{0}}\right)\left(v_{C}-V_{E}\right), \\
S_{0}(x)= & S_{1}(x)-\frac{\alpha}{L}\left(i_{L}-I_{E}\right) v_{C}+\frac{\beta}{C}\left(i_{L}-I_{E}\right) i_{L} \\
& -\frac{\beta}{L}\left(v_{C}-V_{E}\right) v_{C}+\frac{\gamma}{C}\left(v_{C}-V_{E}\right) i_{L}
\end{aligned}
$$

From Eq.s (20a)-(20b) or equivalently from Eq.s (21)-(22), it can be observed that $X_{E}$ is a point of both $S_{0}(x)=0$ and $S_{1}(x)=0$.

Now, the switching strategy (18)-(19) can be interpreted as follows:

$$
\begin{aligned}
& \text { If } u(t)=0 \text { and } S_{0}(x)>0, \text { then } u^{+}=1, \\
& \text { If } u(t)=0 \text { and } S_{0}(x)<0, \text { then } u^{+}=0, \\
& \text { If } u(t)=1 \text { and } S_{1}(x)>0, \text { then } u^{+}=0, \\
& \text { If } u(t)=1 \text { and } S_{1}(x)<0, \text { then } u^{+}=1,
\end{aligned}
$$

where $u^{+}$represent the control signal just after instant $t$, in other words $u^{+}=\lim _{h \rightarrow 0^{+}} u(t+h)$.

Therefore, all trajectories corresponding to $T_{\text {on }}$ interval start from $S_{0}(x)=0$ and end in $S_{1}(x)=0$, where the change to $T_{\text {off }}$ interval takes place. Reciprocally, all trajectories corresponding to $T_{\text {off }}$ interval start from $S_{1}(x)=0$ and end in $S_{0}(x)=0$, where the change to $T_{o n}$ occurs. This behavior is graphically illustrated in Fig. 3. Moreover it has been simulated for different values of parameter $\eta$ (see Fig. 4), $\rho=1000$, and the same set of parameters used in the simulation of Fig. 2. It can be observed that the resulting hysteresis width, i.e. the separation between $S_{0}(x)=0$ and $S_{1}(x)=0$ depends on the value of parameter $\eta$. Namely, for values of $\eta$ near zero the width is maximum and results in slow values of the switching frequency during the initial commutations, while for $\eta$ near 1 the width is minimum and results in very high values of the switching frequency. In 


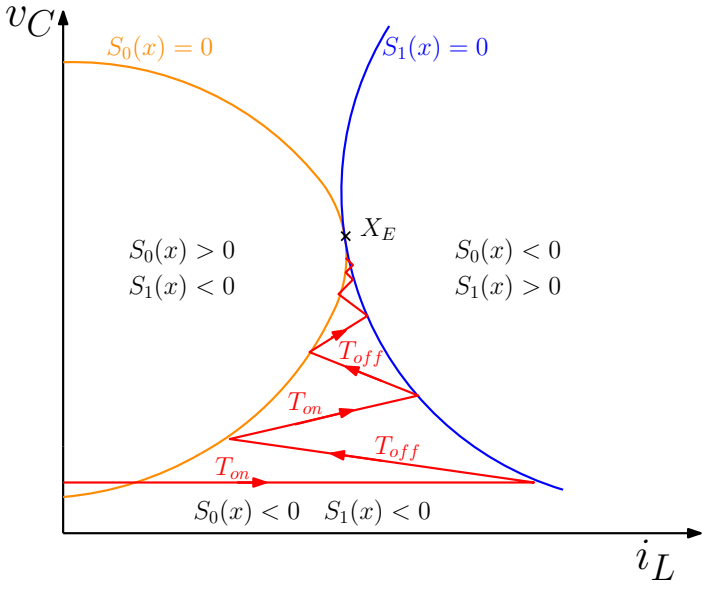

Figure 3. Graphical representation of the state trajectory according the control law (23a)-(23d).
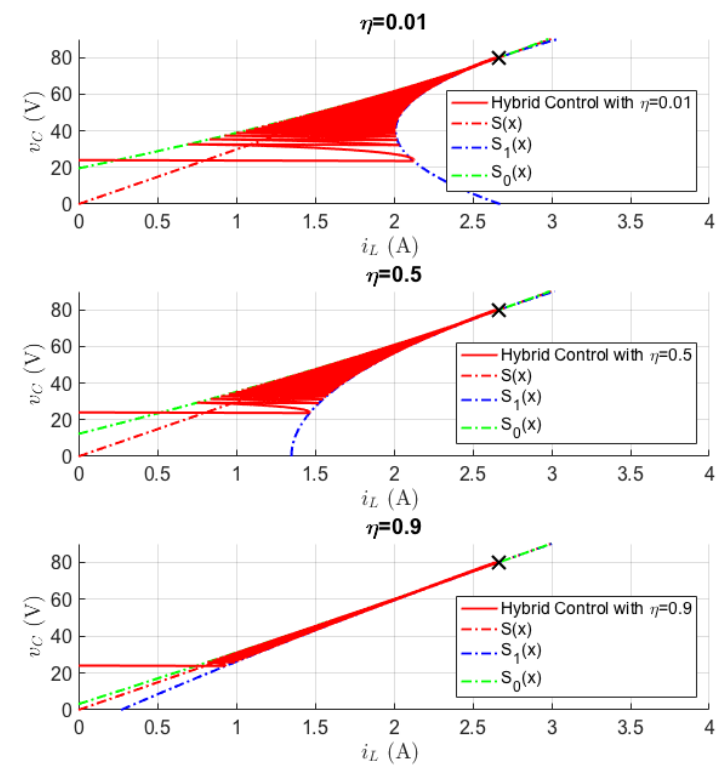

Figure 4. Simulation of the converter start-up in the case of hybrid control, $V_{\text {in }}=24 \mathrm{~V}$ and different values of parameter $\eta$.

all cases the hysteresis width is decreasing as the trajectory approaches the equilibrium point $X_{E}$, where it becomes zero and yields an infinite switching frequency as in the mintype control given by (13). On the other hand, it is worth mentioning that the reduction of initial switching frequency is obtained at the expense of a slight increase of the inrush current since the switching surface $S(x)=0$ of Fig. 2 is located between $S_{0}(x)=0$ and $S_{1}(x)=0$, the two switching surfaces only having in common the equilibrium point $X_{E}$. The increase of inrush current is illustrated in Fig. 5, which reproduces the conditions of the simulations of Fig. 2 for the case of hybrid control and $\eta=0.5$. Note that in the hybrid control the designer has a further degree of freedom (parameter $\eta$ ) in order to manage the number of commutations during transient at the expense of the inrush current.

It is apparent that in both min-type and hybrid control approaches here considered, the switching frequency will be infinite in the equilibrium point. On the other hand, it is well

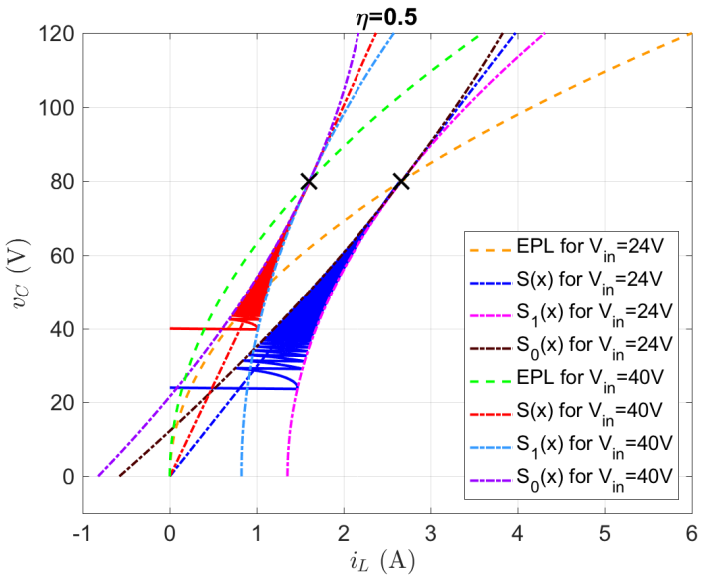

Figure 5. Simulation of the converter start-up for $V_{i n}=24 \mathrm{~V}$ (blue trajectory) and $V_{i n}=40 \mathrm{~V}$ (red trajectory) and hybrid control with $\eta=0.5$.

known that in a real prototype the finite switching frequency imposed by the power devices leads to the existence of ripple in both inductor current and output voltage around its respective equilibrium values $I_{E}$ and $V_{E}$. The presence of ripple in current and voltage yields a limit circle in steady-state around the equilibrium point as depicted in Fig. 6(a), where the straight line represents the trajectory for $T_{o n}$ interval while the parabolic curve corresponds to $T_{\text {off }}$ duration. The closed trajectory evolves between point $A\left(I_{L \min }, V_{C \max }\right)$ and point $B\left(I_{L \max }, V_{C \min }\right)$, where sub-indexes min and max indicate the minimum and maximum value of the corresponding state variable due to the ripple in steady-state. A classical steadystate analysis reveals that the segments of the limit cycle can be expressed as:

$$
v_{C}=V_{C \max }+\frac{L}{C} \frac{I_{E}}{V_{i n}} D^{\prime}\left(I_{L \min }-i_{L}\right) \quad \text { for } T_{o n} \text { interval, }
$$

$$
\begin{aligned}
& v_{C}=V_{C \operatorname{mim}}-\frac{L\left(I_{L \max }-I_{E} D^{\prime}\right)}{C\left(V_{E}-V_{i n}\right)}\left(i_{L}-I_{L \max }\right) \\
& -\frac{L}{2 C\left(V_{E}-V_{i n}\right)}\left(i_{L}-I_{L \max }\right)^{2} \quad \text { for } T_{o f f} \text { interval, }
\end{aligned}
$$

where $I_{E}=\frac{I_{L \max }+I_{L \min }}{2}, V_{E}=I_{E} D^{\prime} R_{0}, D^{\prime}=1-D=1-$ $\frac{T_{o n}}{T}, T_{o n}$ and $T$ being the respective durations of on interval and switching period in steady-state. Defining $\Delta i_{L}=I_{L \max }-$ $I_{L \min }$ and $\Delta v_{C}=V_{C \max }-V_{C \min }$, it can be demonstrated that $V_{E}$ is bounded as shown in Fig. 6(b), where $\Delta i_{L}$ and $\Delta v_{C}$ are given respectively by:

$$
\begin{aligned}
\Delta i_{L} & =\frac{V_{i n}}{L} D T, \\
\Delta v_{C} & =\frac{I_{E}}{C} D D^{\prime} T .
\end{aligned}
$$

Note from (26)-(27) that both horizontal and vertical excursions of the limit cycle are proportional to the switching period $T$. Besides, if $T \rightarrow 0$, then $\Delta i_{L} \rightarrow 0$ and $\Delta v_{C} \rightarrow 0$, or equivalently $I_{L \max }=I_{L \min }=I_{E}$, and $V_{C \max }=V_{C \min }=V_{E}$, which implies that the limit cycle collapses in the equilibrium point $X_{E}$. Therefore, a minimum limit cycle in steady-state 


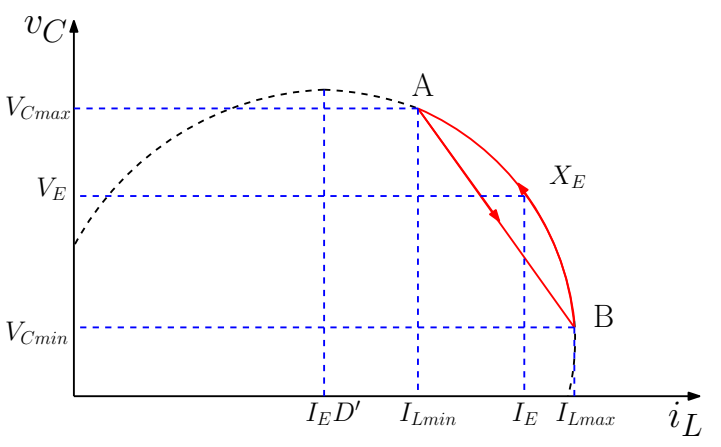

(a)

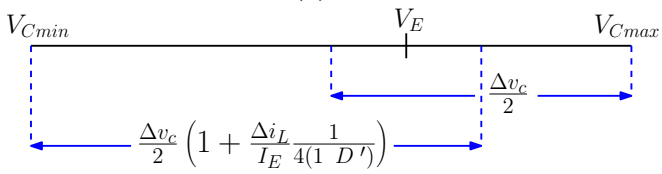

(b)

Figure 6. (a) Limit cycle representing the steady-state converter behaviour, (b) $V_{E}$ bounds.

must be established in the hybrid control approach in order to ensure a proper behavior of the converter. This is equivalent to establish a minimum switching period, whose practical limit should be given by the conditions of a well-designed converter, i.e. the switching frequency must be located at least between one decade or two above the natural frequency $\frac{1}{2 \pi \sqrt{L C}}$.

The above presented steady-state study is particularly important because it allows to better analyze the experimental results given in Section VI. Note that the theoretical results shown in Figs. 4-5 cannot be reproduced at steady-state since it would imply an infinite switching frequency.

The existence of a minimum duration of the switching period in steady-state introduces a new constraint in the hybrid control that affects the stability. This fact has been recently analyzed in [29], where a modification of the hybrid control is considered based on the introduction of a dwell-time. The resulting hybrid system can be formally written as in (16), but the state is extended by an extra state variable $\tau$, which behaves as a timer, so $z=\left[\begin{array}{llll}i_{L} & v_{c} & u & \tau\end{array}\right]^{\top}$, and the flow and jump maps and the flow and jump sets are modified as follows:

$$
\begin{array}{r}
f=\left[\begin{array}{c}
-\frac{R_{L}}{L} i_{L}-\frac{v_{C}}{L}(1-u)+\frac{V_{i n}}{L} \\
-\frac{i_{L}}{C}(1-u)-\frac{1}{C R_{0}} v_{C} \\
0 \\
1
\end{array}\right], \quad g=\left[\begin{array}{c}
i_{L} \\
v_{c} \\
1-u \\
0
\end{array}\right] . \\
\mathcal{C}:=\left\{(x, u, \tau):\left(x-X_{E}\right)^{\top} P\left(A_{u} x+B V_{i n}\right)\right. \\
\left.\leq \eta\left(x-X_{E}\right)^{\top} Q\left(x-X_{E}\right) \wedge \tau \in \mathbb{R}_{+}\right\} \\
\mathcal{D}:=\left\{(x, u, \tau):\left(x-X_{E}\right)^{\top} P\left(A_{u} x+B V_{i n}\right)\right. \\
\left.>\eta\left(x-X_{E}\right)^{\top} Q\left(x-X_{E}\right) \wedge \tau \geq T_{D T}\right\}
\end{array}
$$

where $T_{D T}$ is the dwell-time. This model essentially means that the converter remains in the current topology given by (29a) at least $T_{D T}$ seconds after that the change of topology derived from (29b) has taken place. In [29, Theorem 3] it is proven that the equilibrium point $X_{E}$ is still uniformly globally asymptotically stable despite the introduction of the dwell-time constraint.

Remark 1: Besides the possibility of managing a minimum dwell time, there are other three main advantages of the proposed min-type control strategy with respect to other standard control techniques. The first one is a direct control of the voltage and current trajectories in the phase-plane voltagecurrent (differently from sliding mode control, where only the inductor current is considered in the definition of the sliding surface for the inner loop), this fact allows to obtain noinrush current at start-up, and simultaneously to get a good tracking of the voltage reference, since the trajectories of the system are constrained between $S_{0}$ and $S_{1}$ (See Figs. 4-5). Indeed, in other control techniques, (e.g. sliding mode control, PI control) the inner loop is driven only by the error of the inductor current, without considering the error of the output voltage, which is often controlled by a second loop. Here, in the proposed control technique, the inner loop is driven by the state error $\left(x-X_{E}\right)$, which contains both output voltage and inductor current. Moreover, matrix $P$ is selected by solving the optimization problem (9), leading to the minimization of the cost function $J$. The second advantage is the possibility of choosing the trajectory by selecting the tuning parameter $\eta$, which allows to diminish the distance between $S_{0}$ and $S_{1}$ (See Fig. 6), and hence directly to increase the number of commutations. The latter increment of the switching frequency diminishes the ripple of the inductor current. Finally, the use of the hybrid formalism [22] provides a natural framework for this class of systems, where both continuous-time and discretetime dynamics coexist, ensuring the stability of the system.

\section{EXPERIMENTAL SET-UP}

A test setup has been suitably built to validate the proposed control technique. The picture of the test bench is shown in Fig. 7. The general architecture of the experimental set-up is shown in Fig. 8. The converter under test is shown in Fig. 1. The nominal power is $60 \mathrm{~W}$ and the components used for the experimental prototype are:

- Inductor $L$ : AGP4233-473ME;

- Capacitor $C$ : MKP1848620094P4;

- Switches $S-\bar{S}$ : SiC MOSFET C3M0065090D;

- Auxiliary diode D: 8TQ100S.

The passive elements have the same nominal values used in the simulations in Figs. 2-5. The MOSFETs are sourced by means of a power driver MGJ2D121505SC, which provides the right amount of current for the turning-on and turningoff of the components. Moreover, in order to guarantee a galvanic isolation between the signal part and the power part of the circuit, two optocouplers ADUM3401CRWZ have been used. The controller has been implemented digitally using the TI microcontroller TMS320F28379D as shown in the block diagram of Fig. 8. The inductor current is measured by means of an Hall-effect sensor LEM LTS-15-NP, while the output voltage is measured by means of a cascade between a voltage divider and an operational amplifier LM324 in buffer configuration. Both signals are sampled at $1.5 \mathrm{MHz}$ by the respective analog to digital converters to be subsequently 


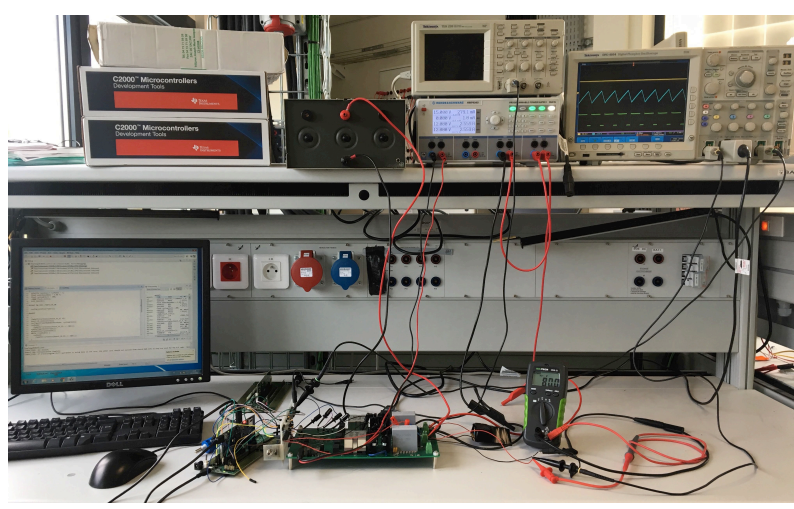

Figure 7. Test bench.

Table II

LOGIC TABLE OF THE COMMUTATIONS

\begin{tabular}{lll||l}
$u\left(k T_{s a}\right)$ & $S_{u}\left(x\left(u\left(k T_{s a}\right)\right)<0\right.$ & $\tau \geq T_{D T}$ & $\begin{array}{l}\text { Output } \\
u\left((k+1) T_{s a}\right)\end{array}$ \\
\hline 0 & 0 & 0 & 0 \\
0 & 0 & 1 & 1 \\
0 & 1 & 0 & 0 \\
0 & 1 & 1 & 0 \\
1 & 0 & 0 & 1 \\
1 & 0 & 1 & 0 \\
1 & 1 & 0 & 1 \\
1 & 1 & 1 & 1 \\
\hline
\end{tabular}

processed by the microcontroller. The latter uses the MatlabSimulink ${ }^{\circledR}$ information developed in the simulation described above to generate the control signal $u$. In particular, it provides the coordinates of the equilibrium point $X_{E}$ for a desired value of the output voltage $V_{E}$ and input voltage, and the sampled values of $S_{0}(x)$ and $S_{1}(x)$ for the mentioned sampling frequency. Subsequently, the proposed control law is digitalized by considering Table II, where $\frac{1}{T_{S a}}=1.5 \mathrm{MHz}$ is the sampling frequency. Table II gives the following logic relation:

$$
\begin{array}{r}
u\left((k+1) T_{s a}\right)=\left(\neg u\left(k T_{s a}\right) \wedge \neg s\left(k T_{s a}\right) \wedge t\left(k T_{s a}\right)\right) \\
\vee\left(u\left(k T_{s a}\right) \wedge \neg s\left(k T_{s a}\right) \wedge \neg t\left(k T_{s a}\right)\right) \\
\vee\left(u\left(k T_{s a}\right) \wedge s\left(k T_{s a}\right)\right),
\end{array}
$$

where $s\left(k T_{s a}\right)$ and $t\left(k T_{s a}\right)$ represent the logic status respectively of relations $S_{u}\left(x\left(u\left(k T_{s a}\right)\right)<0\right.$ and $\tau \geq T_{D T}$.

A dwell-time $T_{D T}$ of $3 \mu \mathrm{s}$ has been considered, which ensures a minimum switching period of $5 \mu \mathrm{s}$ and results in a maximum switching period of $10 \mu \mathrm{s}$. As a consequence, the control input $u\left(k T_{S a}\right)$ calculated according to (30) results in a two-level square signal with a switching frequency ranging from $100 \mathrm{kHz}$ to $200 \mathrm{kHz}$. This internal signal of the microcontroller is compared with a $10 \mathrm{MHz}$ periodic triangular internal signal of the PWM module to obtain the signals $u$ and $\bar{u}$ that activate the optocouplers and hence the power switch. The high level of signal $u\left(k T_{S a}\right)$, representing the binary value 1 , is always higher than the periodic triangular signal, so that the comparison will always yield a binary value 1 at the output of the PWM during the interval of time in which $u\left(k T_{S a}\right)=1$. On the contrary, the low level of $u\left(k T_{S a}\right)$,

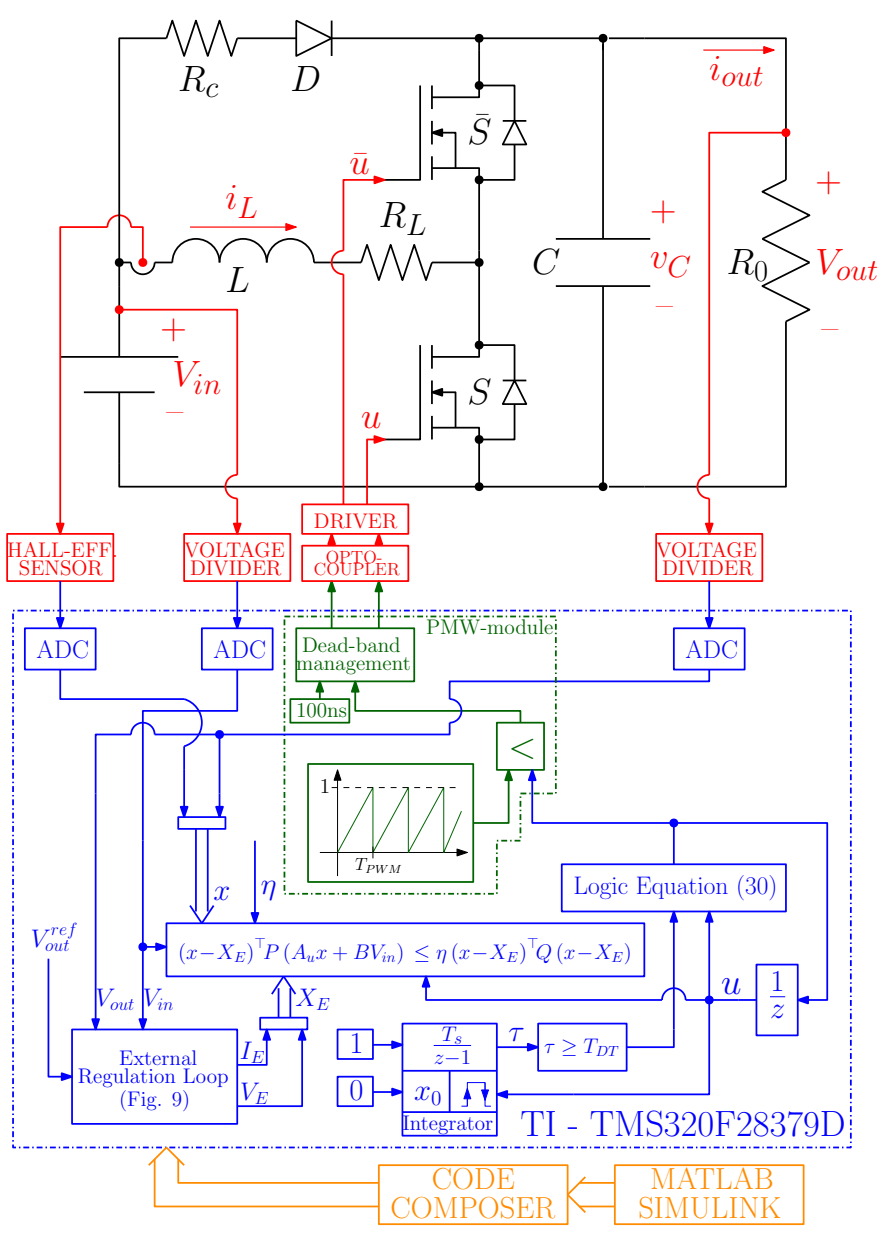

Figure 8. Block diagram of the experimental set-up.

representing the binary value 0 , is always below the periodic triangular signal, which leads to a binary value 0 at the output of the PWM. Thus, the output of the PWM reproduces the variable switching frequency signal generated by the min-type control strategy and provides the necessary level of current to activate the optocouplers. The PWM frequency (which is constant and equal to $1 / T_{P W M}$ ) has been chosen high enough in order to reduce possible static errors due to the fact that the proposed min-type control strategy generates a variable switching frequency that will be an integer multiple of the PWM frequency. Finally, a dead-band of 100ns has been considered in order to avoid the simultaneous conduction of $S$ and $\bar{S}$. This mechanism is represented in Fig. 8 .

In order to make the system robust against input voltage variations as well as load variations, a PI corrector processing the output voltage error has been added to modify the equilibrium point $\left(V_{E}, I_{E}\right)$. As shown in Fig. 9, the PI controller processes the output voltage error and gives a reference value of $V_{E}$. By using this value of $V_{E}$ and the measured value of the input voltage, the equilibrium value of the current is computed by means of Eq. (2). Finally, the values of $V_{E}, I_{E}$ and the measured value of $V_{i n}$ are used by the proposed mintype control algorithm in order to solve the arg-min problem shown in Eq. (4). As a result, the system is robust against variations of the input voltage as well as load variations. 


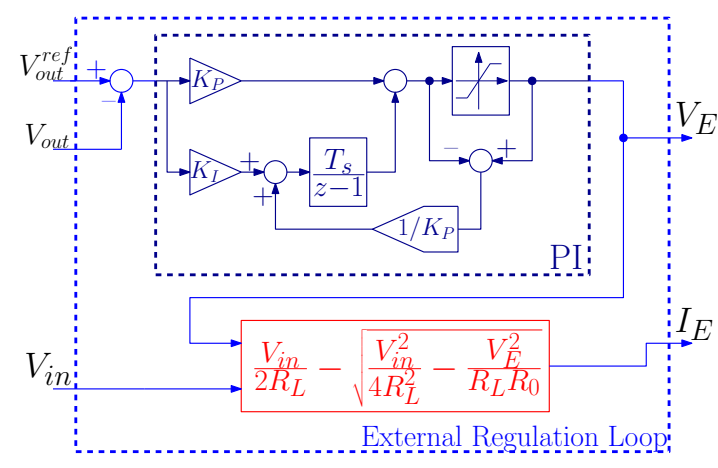

Figure 9. Block diagram of the external regulation loop.

Details about parameter design of the PI are omitted here due to space limitations.

Note that the proposed control technique does not depend on the particular hardware used for the implementation, therefore the use of a FPGA represents also a good solution instead of a microcontroller, especially when higher switching frequencies are considered.

\section{EXPERIMENTAL RESULTS}

Fig. 10 show the waveforms of the output voltage and of the inductor current during a start-up, where the initial instant in the oscilloscope capture corresponds to $0.3 \mathrm{~ms}$, by means of the proposed min-type strategy. It can be observed that the converter attains the desired equilibrium point corresponding to the output voltage coordinate $V_{E}=80 \mathrm{~V}$ for an input voltage $V_{i n}=24 \mathrm{~V}$. Moreover, no inrush current is shown even after a fast transient state. In order to highlight the behavior of the min-type strategy implemented by the proposed controller, a zoom of the capacitor voltage and the corresponding control input $u$ during the start-up is shown in Fig. 11. Note that both Fig. 10 and Fig. 11 have been obtained by selecting $\eta=0.5$. Fig. 11 confirms the theoretical predictions, and it is perfectly coherent with the simulation results of Section IV.

The resulting waveforms of the output voltage and of the inductor current in steady-state are shown in Fig. 12, where it can be appreciated an average value about $I_{E}=2.75 \mathrm{~A}$, a current ripple $\Delta i_{L}=3 \mathrm{~A}$, and a switching frequency of about $150 \mathrm{kHz}$. The steady-state waveforms shown in the paper correspond to an output power of about $65 \mathrm{~W}$. Note that the topology of the boost converter considered in this paper is bidirectional and never enters in discontinuousconduction mode because the inductor current can be also negative, thus the high ripple is not a drawback. However, it is possible to reduce the ripple by decreasing the dwell time $T_{D T}$ (at steady-state), or by acting on the design parameter $\eta$ (during transient). It has been experimentally noted that the switching frequency, in steady-state, is about $1 /\left(2 \lambda T_{D T}\right)$, where $\lambda \in[1,1.5]$, and this frequency is maintained almost constant despite the equilibrium point changes. For example, $T_{D T}=3 \mu s$ results in a switching frequency of about $150 \mathrm{kHz}$ regardless of the equilibrium point. Hence, the above given empirical rule can be used for sizing the passive components of the converter such as EMI flters, reactive components, etc.
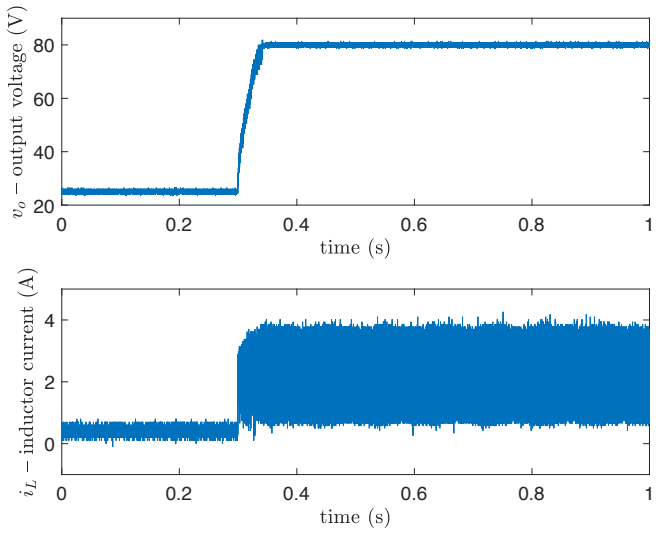

Figure 10. Output voltage and inductor current during a start-up test by means of the proposed min-type control strategy.
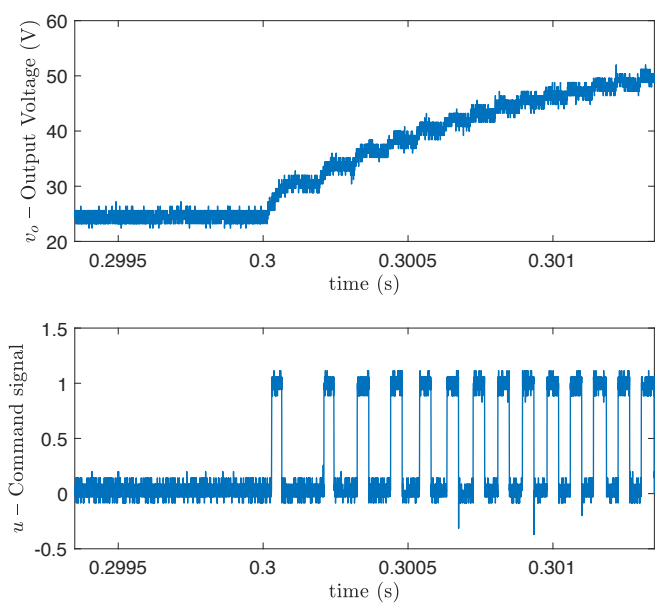

Figure 11. Zoom of the output voltage (a) and corresponding control input $u$ (b), during a start-up test by means of the proposed min-type control strategy.

Therefore, the proposed approach is in a clear-cut contrast with conventional hysteresis-based controllers, where the switching frequency in steady-state depends on both load resistance and input voltage. Thus, the proposed strategy combines the fast transient response of a hysteresis controller with variable switching frequency with the predictable PWM behavior in steady-state with constant switching frequency.

In order to evaluate the steady-state performance, Fig. 13 depicts the measured system efficiency for different values of input power. It can be observed a good efficiency, about 94\%, under nominal operating conditions.

In order to compare the proposed control strategy with other control solutions, the start-up in the respective case of a SM and a PI controller are shown under the same operating conditions. In particular, Figs. 14 and 15 show the waveforms of the output voltage and of the inductor current during the start-up by means of a SM control strategy and a PI control strategy respectively. By comparing Figs. 14 and 15 with Fig. 10 it is shown that with the proposed control strategy a better transient can be obtained in terms of settling-time as well as in dynamic precision. Indeed, it can be noted a higher settling-time if the PI controller is used, and a lower dynamic 

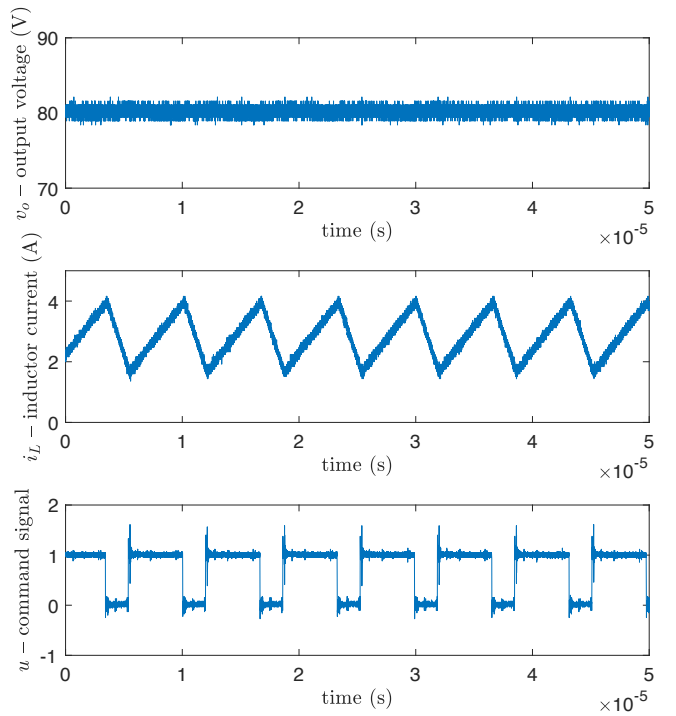

Figure 12. Output voltage, inductor current and control signal at steadystate by means of the proposed min-type control strategy.

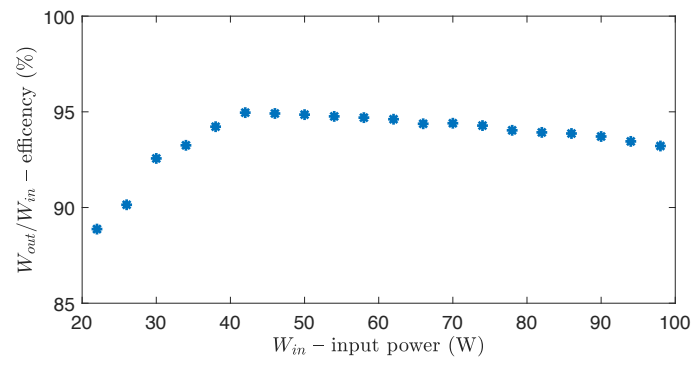

Figure 13. Efficiency of the system at steady-state for different values of input power.

precision during transient time if the SM controller is used. This is due to the fact that, with the proposed solution, a direct control of both voltage and current trajectories in the phaseplane voltage-current is obtained, differently from sliding mode control where only the inductor current is considered in the definition of the sliding surface. Moreover, no-inrush current is shown at start-up together a suited tracking of the voltage reference.

It has to be pointed out that the proposed min-type control strategy can be also used to reject both input voltage and load perturbations by a simple insertion of an appropriate corrector processing the output voltage error as shown in Section V. Fig. 16.(a) show the converter response for an input voltage variation from $24 \mathrm{~V}$ to $29 \mathrm{~V}$ and from $29 \mathrm{~V}$ to $24 \mathrm{~V}$. Note that the output voltage recovers the desired value of $80 \mathrm{~V}$ after a fast transient and a relatively low overshoot while the increment of energy introduced by the input voltage variation is appropriately absorbed by the inductor current. Under the same conditions, the SM controller and the PI controller have been tested and the results are shown in Figs. 16.(b) and 16.(c) respectively. Also in this case, with the min-type strategy a lower settling time and a higher dynamic precision is obtained.

Similarly, Fig. 17.(a) depicts the converter response to a step variation in the load resistance from $100 \Omega$ to $150 \Omega$ and
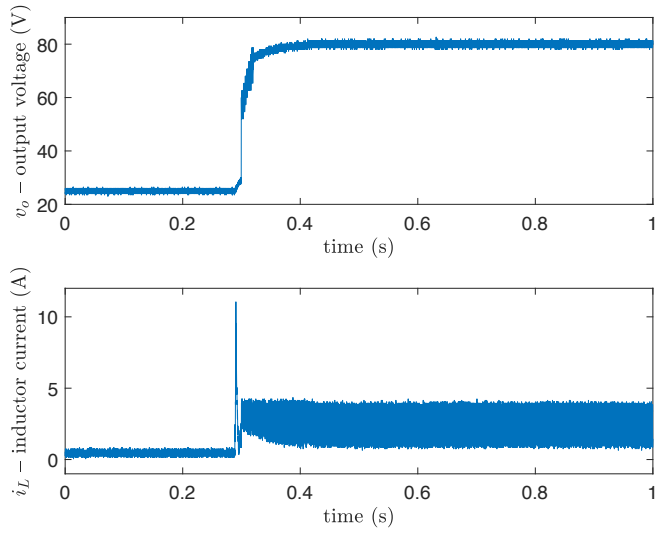

Figure 14. Output voltage (a) and inductor current (b) during a start-up test by means of SM control strategy.
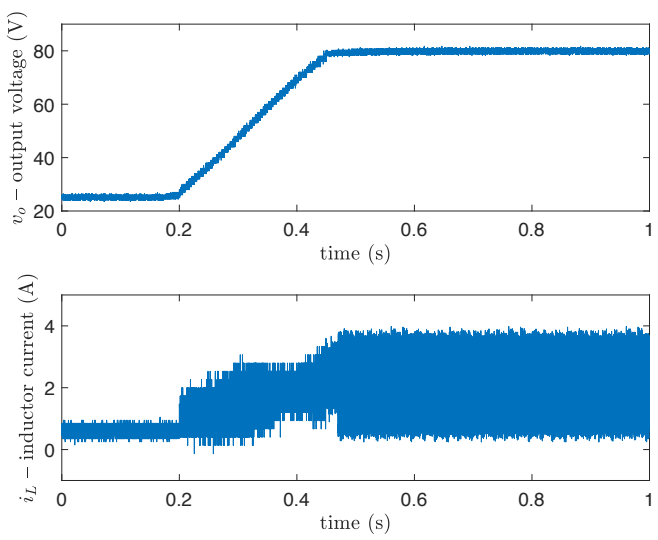

Figure 15. Output voltage (a) and inductor current (b) during a start-up test by means of PI control strategy.

from $150 \Omega$ to $100 \Omega$. It can be observed the fast output voltage recovery and the adjustment of the inductor current to the new current demand at the output port. The results obtained with the SM controller and the PI controller under the same variations of the output load are shown in Figs. 17.(b) and 17.(c) respectively. Likewise, the min-type control strategy exhibits a better tracking of the desired trajectory and a faster output voltage recovery.

Finally, in order to test the proposed min-type control strategy in very challenging operating conditions, an experimental test with a large variation of the input voltage, from $48 \mathrm{~V}$ to $24 \mathrm{~V}$ is shown in Fig. 18. Moreover an experimental test of a load resistance variation, from no load to $100 \Omega$, is shown in Fig. 19. In both cases, suited performances (in terms of fast output voltage recovery and good transient behavior of the inductor current) of the min-type control strategy is shown.

\section{CONCLUSIONS}

An accurate analytical model of a min-type control strategy based on a nonlinear switching surface has been constructed for a synchronous boost converter and interpreted under the perspective of sliding-mode control. The requirements of finite switching frequency for the power devices has led to a modification of the control approach by establishing a hybrid 

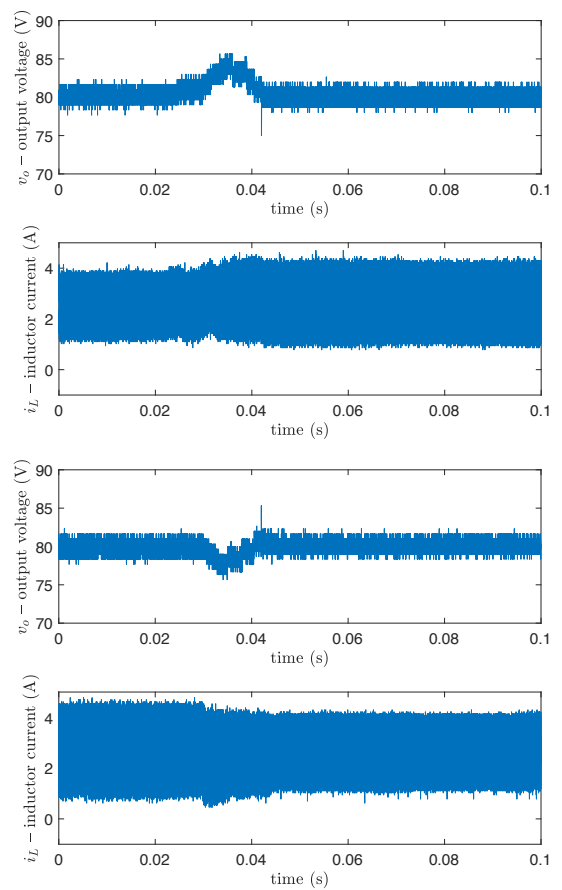

(a)
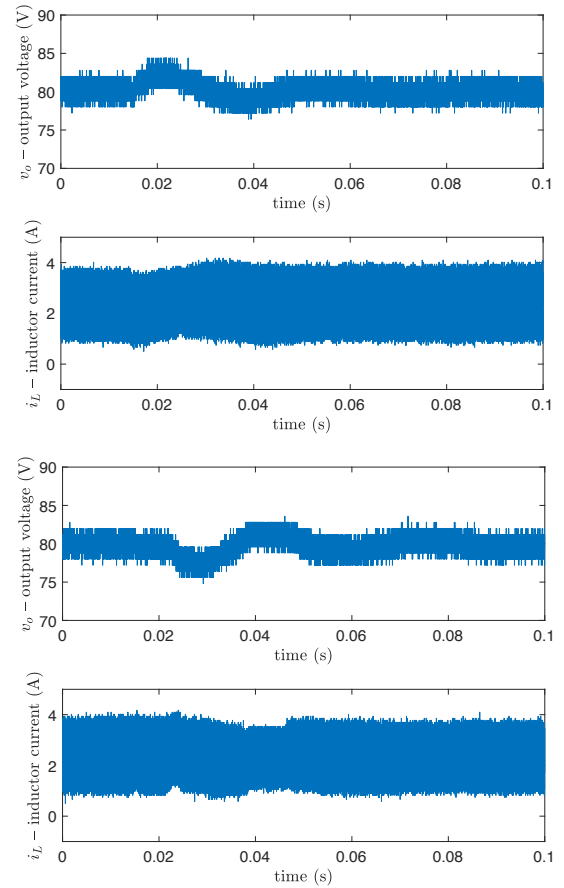

(b)
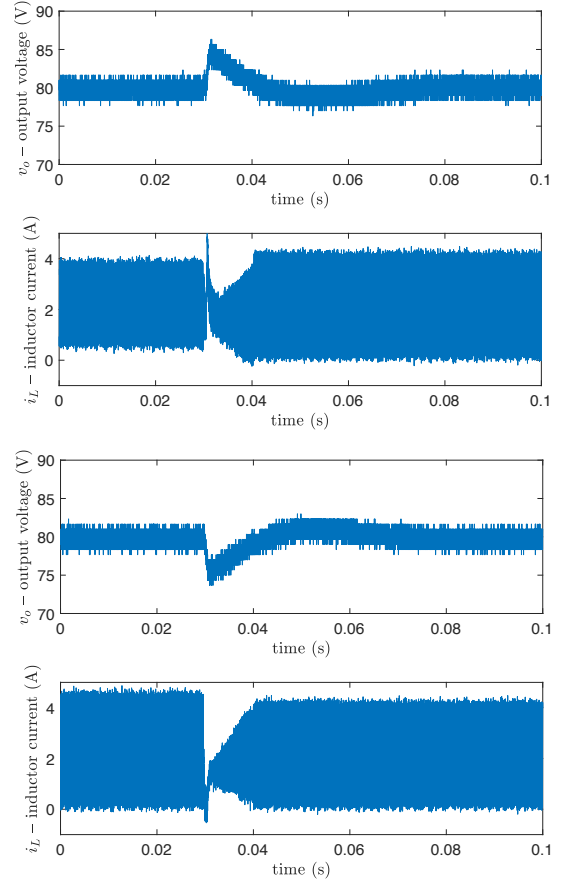

(c)

Figure 16. Output voltages and inductor currents with an additional outer regulation loop during a step variation in the input voltage from $24 \mathrm{~V}$ to $29 \mathrm{~V}$ (upper plots), and from $29 \mathrm{~V}$ to $24 \mathrm{~V}$ (bottom plots) by using: (a) proposed min-type control strategy, (b) SM controller and (c) PI controller.
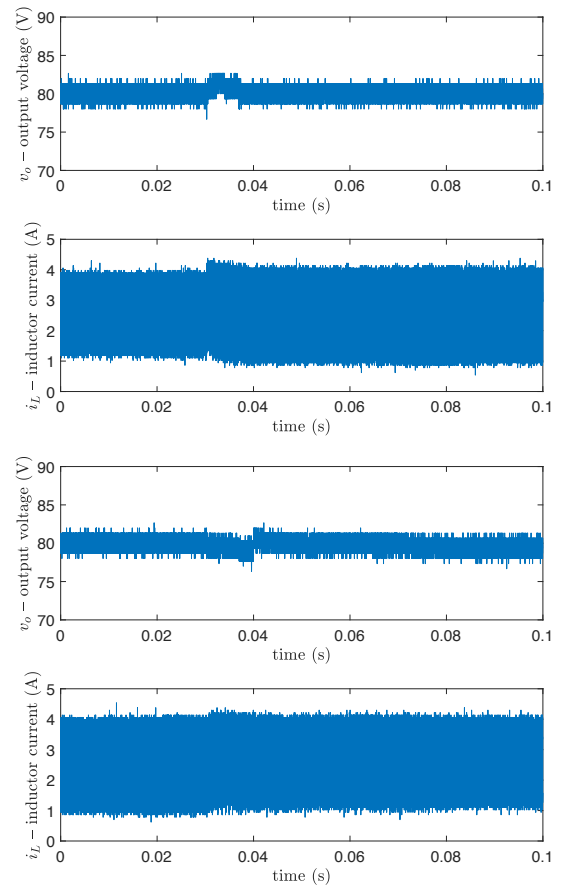

(a)
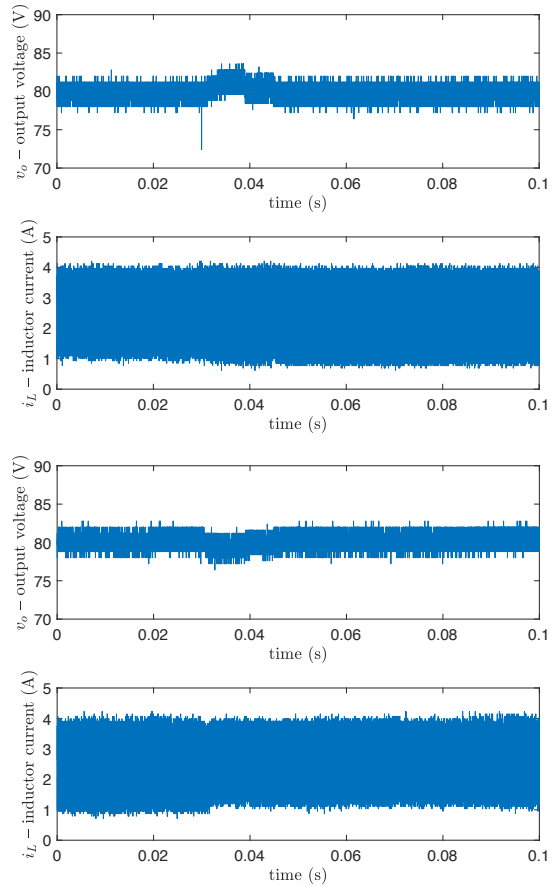

(b)
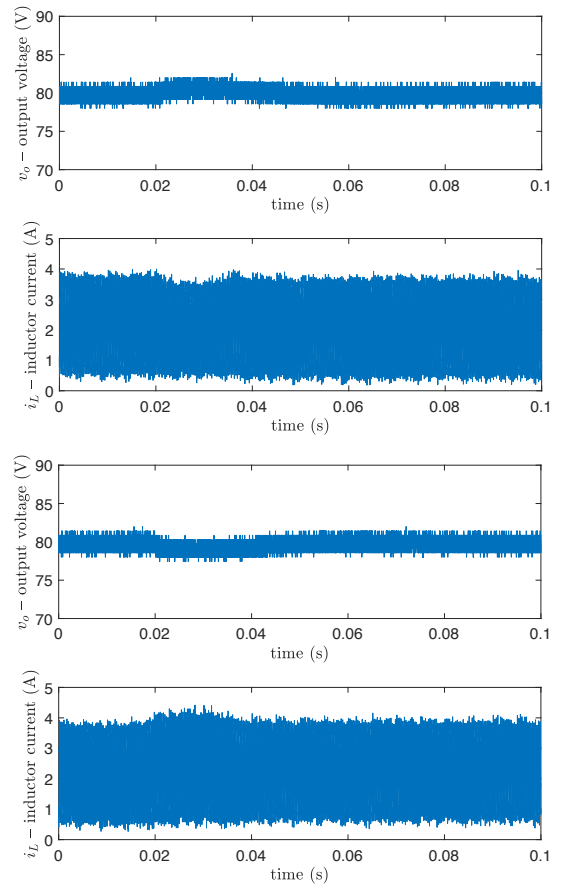

(c)

Figure 17. Output voltages and inductor currents with an additional outer regulation loop during a step variation in the load resistance from $100 \Omega$ to $150 \Omega$ (upper plots), and from $150 \Omega$ to $100 \Omega$ (bottom plots) by using: (a) proposed min-type control strategy, (b) SM controller and (c) PI controller. 

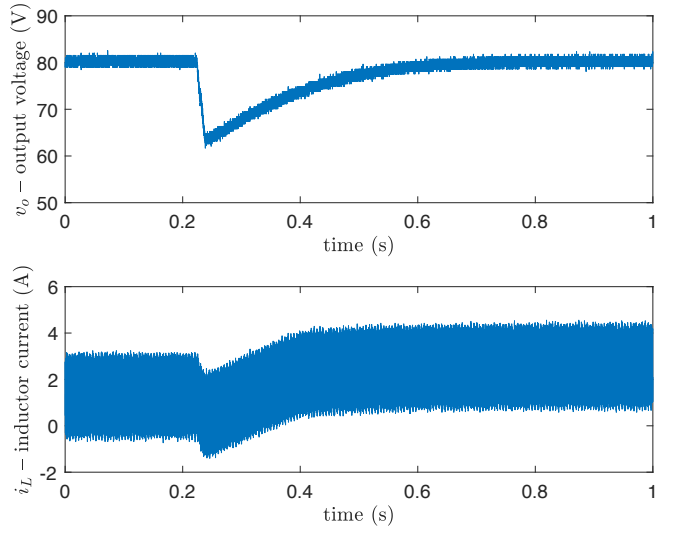

Figure 18. Output voltages and inductor currents with an additional outer regulation loop during a step variation in the input voltage from $48 \mathrm{~V}$ to $24 \mathrm{~V}$ with the proposed min-type control strategy.
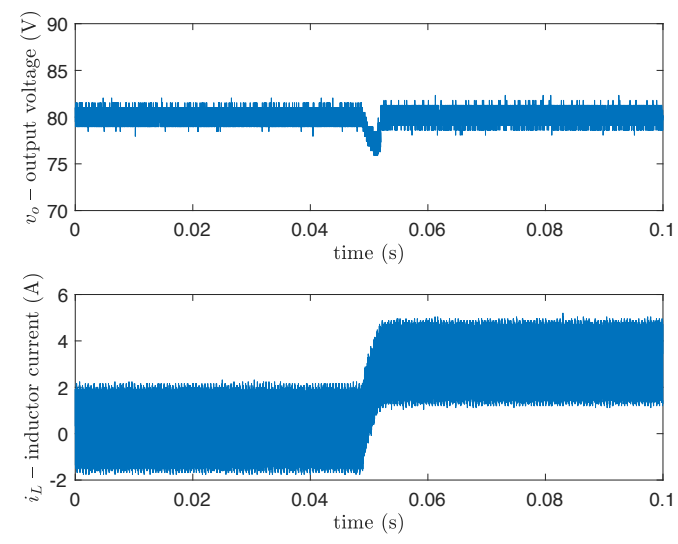

Figure 19. Output voltages and inductor currents with an additional outer regulation loop during a step variation in the load resistance from noload to $100 \Omega$ with the proposed min-type control strategy.

strategy that differentiates the dynamics of continuity in the current converter topology from the dynamics of topology change. This modification has had an immediate theoretical effect on the switching frequency during the startup because it has introduced a hysteresis width, which decreases from its maximum value corresponding to the first $T_{\text {on }}$ trajectory until zero when the equilibrium point is attained. The infinite switching frequency that would result in steady-state has been precluded by including a dwell-time constraint in the hybrid control, which eventually results in an upper bound for the switching frequency. A prototype has been constructed to verify the theoretical predictions. The control algorithm has been implemented by means of a microprocessor that processes the samples of inductor current and capacitor voltage to provide the control signal, which is adapted at the microcontroller output to the optocouplers by means of the PWM module. The desired equilibrium point has been reached after a very fast transient and no inrush current has been observed. The addition of a PI to compensate for input voltage and load variations ensures the output voltage regulation.

\section{REFERENCES}

[1] R. W. Erickson and D. Maksimovic, Fundamentals of power electronics. Springer Science \& Business Media, 2007.

[2] UC3842/3/4/5 provides low-cost current-mode control. Unitrode application note U-100A, Texas Instruments Incorporated, 1999.

[3] R. Redl and N. O. Sokal, "Current-mode control, five different types, used with the three basic classes of power converters: small-signal ac and large-signal dc characterization, stability requirements, and implementation of practical circuits," in Power Electronics Specialists Conference. IEEE, 1985, pp. 771-785.

[4] Y. Qiu, X. Chen, and H. Liu, "Digital average current-mode control using current estimation and capacitor charge balance principle for DC-DC converters operating in DCM," IEEE Transactions on Power Electronics, vol. 25, no. 6, pp. 1537-1545, 2010.

[5] J. Chen, A. Prodic, R. W. Erickson, and D. Maksimovic, "Predictive digital current programmed control," IEEE Transactions on Power Electronics, vol. 18, no. 1, pp. 411-419, 2003.

[6] C. Carrejo, E. Vidal-Idiarte, R. Giral, and L. Martinez-Salamero, "Predictive digital interpolation current control for DC-DC power converters," IET Power Electronics, vol. 2, no. 5, pp. 545-554, 2009.

[7] P. Athalye, D. Maksimovic, and R. Erickson, "Variable-frequency predictive digital current mode control," IEEE Power Electronics Letters, vol. 2, no. 4, pp. 113-116, 2004.

[8] E. Vidal-Idiarte, C. E. Carrejo, J. Calvente, and L. Martínez-Salamero, "Two-loop digital sliding mode control of DC-DC power converters based on predictive interpolation," IEEE Transactions on Industrial Electronics, vol. 58, no. 6, pp. 2491-2501, 2011.

[9] K.-Y. Cheng, F. Yu, F. C. Lee, and P. Mattavelli, "Digital enhanced $v^{2}$ type constant on-time control using inductor current ramp estimation for a buck converter with low-ESR capacitors," IEEE Transactions on Power Electronics, vol. 28, no. 3, pp. 1241-1252, 2013.

[10] G. Zhou and J. Xu, "Digital average current controlled switching DCDC converters with single-edge modulation," IEEE Transactions on Power Electronics, vol. 25, no. 3, pp. 786-793, 2010.

[11] Y.-S. Lai and C.-A. Yeh, "Predictive digital-controlled converter with peak current-mode control and leading-edge modulation," IEEE Transactions on Industrial Electronics, vol. 56, no. 6, pp. 1854-1863, 2009.

[12] G. Zhou, J. Xu, and Y. Jin, "Improved digital peak current predictive control for switching DC-DC converters," IET power electronics, vol. 4 , no. 2, pp. 227-234, 2011.

[13] W. Stefanutti and P. Mattavelli, "Fully digital hysteresis modulation with switching-time prediction," IEEE Transactions on Industry Applications, vol. 42, no. 3, pp. 763-769, 2006.

[14] E. Vidal-Idiarte, A. Marcos-Pastor, G. Garcia, A. Cid-Pastor, and L. Martinez-Salamero, "Discrete-time sliding-mode-based digital pulse width modulation control of a boost converter," IET Power Electronics, vol. 8, no. 5, pp. 708-714, 2015.

[15] G. S. Deaecto, J. C. Geromel, F. Garcia, and J. Pomilio, "Switched affine systems control design with application to DC-DC converters," IET control theory \& applications, vol. 4, no. 7, pp. 1201-1210, 2010.

[16] J. C. Geromel, G. S. Deaecto, and J. Daafouz, "Suboptimal switching control consistency analysis for switched linear systems," IEEE Transactions on Automatic Control, vol. 58, no. 7, pp. 1857-1861, 2013.

[17] G. S. Deaecto, "Dynamic output feedback $\mathcal{H}_{\infty}$ control of continuoustime switched affine systems," Automatica, vol. 71, pp. 44-49, 2016.

[18] L. Martinez-Salamero, G. García, M. Orellana, C. Lahore, B. Estibals, C. Alonso, and C. E. Carrejo, "Analysis and design of a sliding-mode strategy for start-up control and voltage regulation in a buck converter," IET Power Electronics, vol. 6, no. 1, pp. 52-59, 2013.

[19] G. S. Deaecto, M. Souza, and J. C. Geromel, "Chattering free control of continuous-time switched linear systems," IET Control Theory \& Applications, vol. 8, no. 5, pp. 348-354, 2014.

[20] C. Duan and F. Wu, "Analysis and control of switched linear systems via dwell-time min-switching," Systems \& Control Letters, vol. 70, pp. 8-16, 2014.

[21] C. Albea-Sanchez, G. Garcia, and L. Zaccarian, "Hybrid dynamic modeling and control of switched affine systems: application to DCDC converters," in 54th Annual Conference on Decision and Control. IEEE, 2015, pp. 2264-2269.

[22] R. Goebel, R. G. Sanfelice, and A. R. Teel, Hybrid Dynamical Systems: modeling, stability, and robustness. Princeton University Press, 2012.

[23] T. A. Theunisse, J. Chai, R. G. Sanfelice, and W. M. H. Heemels, "Robust global stabilization of the DC-DC boost converter via hybrid control," IEEE Transactions on Circuits and Systems I: Regular Papers, vol. 62, no. 4, pp. 1052-1061, 2015. 
[24] L. Martínez-Salamero, G. García, M. Orellana, C. Lahore, and B. Estibals, "Start-up control and voltage regulation in a boost converter under sliding-mode operation," IEEE Transactions on industrial electronics, vol. 60, no. 10, pp. 4637-4649, 2013.

[25] J. Lofberg, "YALMIP: A toolbox for modeling and optimization in MATLAB," in International Symposium on Computer Aided Control Systems Design. IEEE, 2004, pp. 284-289.

[26] V. Utkin, J. Guldner, and J. Shi, Sliding mode control in electromechanical systems. CRC press, 2009.

[27] L. Martinez-Salamero, A. Cid-Pastor, A. El Aroudi, R. Giral, J. Calvente, and G. Ruiz-Magaz, "Sliding-mode control of DC-DC switching converters," in 18th IFAC World Congress. IFAC, 2011, pp. 1910-1916.

[28] J. M. Bosque-Moncusi, H. Valderrama-Blavi, F. Flores-Bahamonde, E. Vidal-Idiarte, and L. Martínez-Salamero, "Using low-cost microcontrollers to implement variable hysteresis-width comparators for switching power converters," IET Power Electronics, vol. 11, no. 5, pp. 787795, 2017.

[29] C. Albea-Sanchez, G. García, S. Hadjeras, M. Heemels, and L. Zaccarian, "Practical stabilisation of switched affine systems with dwell-time guarantees," Submitted to IEEE Transactions on Automatic Control, 2018. [Online]. Available: https://hal.archives-ouvertes.fr/hal01617999/document

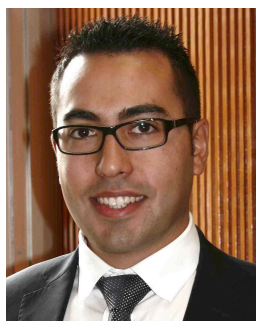

Antonino Sferlazza (S'12-M'15) was born in Palermo, Italy, in 1987. He received the master?s degree in automation engineering and the Ph.D. degree in mathematics and automation from the University of Palermo, Palermo, Italy, in 2011 and 2015, respectively. In 2013, he was visiting Ph.D. student at University of California, Santa Barbara, CA, USA, in the field of modeling and analysis of stochastic hybrid systems. From 2016 to 2017, he was a Junior Researcher with the University of Palermo. From 2017 to 2018, he was Researcher with Laboratory for Analysis and Architecture of Systems, Toulouse, France, working in the field of control of power converter. He is currently a Researcher in systems and control engineering with the University of Palermo. His research interests include the development of feedback control algorithms for nonlinear dynamical systems, optimization techniques, estimation of stochastic systems, and applications of control of electrical drives and power converters.

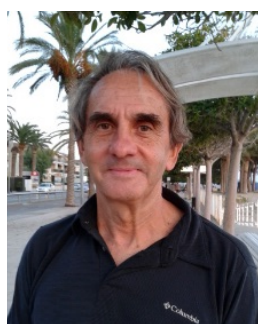

Luis Martínez-Salamero (M'79-SM'86) received the Ingeniero de Telecomunicacin degree in 1978 and the Ph.D. degree in 1984, both at the Universidad Politcnica de Catalua, Barcelona, Spain. From 1978 to 1992, he taught circuit theory, analog electronics and power processing at the Escuela Tcnica Superior de Ingenieros de Telecomunicacin , Barcelona, Spain. From 1992 to 1993, he was a visiting professor at the Center for Solid State Power Conditioning and Control, Department of Electrical Engineering, Duke University, Durham, NC. From 2003 to 2004, 2010 to 2011, and March-September 2018 he was a visiting scholar at the Laboratory of Architecture and Systems Analysis (LAAS), National Agency for Scientific Research (CNRS), Toulouse, France. Since 1995 he has been a full professor with the Department of Electrical Electronic and Automatic Control Engineering, School of Electrical and Computer Engineering, Rovira i Virgili University, Tarragona, Spain, where he managed the Research Group in Automatic Control and Industrial Electronics (GAEI) in the period 1998-2018. His research interests include structure and control of power conditioning systems, namely, electrical architecture of satellites and electric vehicles, as well as nonlinear control of converters and drives, and power conditioning for renewable energy.

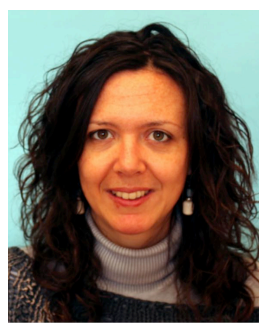

Carolina Albea-Sanchez received her $\mathrm{PhD}$ in automatic control in 2010 from the University of Sevilla, Spain, and the University of Grenoble, France. From 2010 to 2011, she held a post-doctoral position at the CEA-LETI Minatec campus in Grenoble, France, on the control of nanoelectronic circuits. Then in 2011 she became Associate Professor at the University of Toulouse III (Université Paul Sabatier). Her research is performed at the LAAS-CNRS. Her topics of interest cover hybrid control of switched systems, hybrid dynamical systems, control of electronic converters.

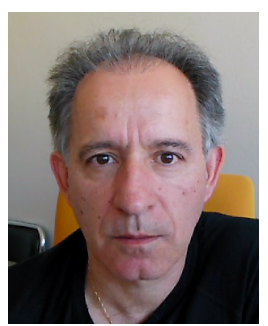

Germain García received the M.S. degree in electrical engineering and computer sciences from Institut National des Sciences appliquees (INSA), Toulouse, France, in 1984, the Ph.D degree in 1988 and the Habilitation Diriger des Recherches in 1997. Currently he is Professor in the department of Electrical Engineering and Computer Sciences at INSA and researcher at LAAS-CNRS, Toulouse, France. His research interests are robust and nonlinear control, singularly perturbed systems and control of systems with bounded actuators. More recently, his research interests focuses on hybrid and switching control techniques applied to the control of power converters.

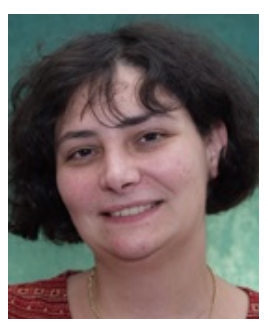

Corinne Alonso is currently Full Professor at the Department of Electrical, Electronic and Automatic Engineering of the University of Paul Sabatier, Toulouse and Researcher at LAASCNRS lab. She holds a PhD in Electrical Engineering from the Institut National Polytechnique of Toulouse, a HDR (Habilitation diriger les Recherches) and a Master's Degree in Electrical Engineering and Electrical Engineering from the University of Paul Sabatier, Toulouse. She has more than 25 years of academic and professional experience devoted to power electronics design and its modelling dedicated to embedded and new grids, renewable and intermittence energy sources and their impact on micro-grids. 